\title{
Transcriptional Activation of Putative Calmodulin Genes Am-cam-1 and Am-cam-2 from Aquilaria microcarpa, in Response to External Stimuli
}

\author{
Yoshihide Kenmotsu, ${ }^{a}$ Yoshimi Yamamura, ${ }^{a}$ Shinjiro Ogita, ${ }^{b}$ Yasuo Katoh, ${ }^{b}$ and \\ Fumiya KurosAKI ${ }^{*}, a$ \\ ${ }^{a}$ Laboratory of Plant Resource Sciences, Graduate School of Medicine and Pharmaceutical Sciences for Research, \\ University of Toyama; Sugitani, Toyama 930-0194, Japan: and ${ }^{b}$ Laboratory of Plant and Cell Engineering, Graduate \\ School of Engineering, Toyama Prefectural University; Kurokawa, Imizu 939-0398, Japan.
}

Received May 27, 2010; accepted August 12, 2010; published online August 16, 2010

\begin{abstract}
A homology-based cloning strategy yielded two cDNA clones designated $A m-c a m-1$ and $A m-c a m-2$, presumably encoding calmodulin protein from a callus culture derived from the leaf tissues of Aquilaria microcarpa. An appreciable increase in the transcriptional activity of $\mathrm{Am}$-cam-1 was reproducibly observed by exposure of the cell culture to methyl jasmonate, as analyzed by a reverse-transcription polymerase chain reaction. The expression level of the gene also increased when the cells were treated with yeast extract. The transcription of $\mathrm{Am}$-cam2 was similarly stimulated by the treatment with methyl jasmonate and yeast extract, however, the intensities of the enhanced expression appeared to be lower as compared with that of $\mathrm{Am}$-cam-1. In contrast, $\mathrm{Ca}^{2+}$-ionophore A23187 did not show inducing activity for the expression of these two calmodulin genes. These results suggest that $A m-c a m-1$ and $A m-c a m-2$ and their products play important roles in signal transduction processes in methyl jasmonate- and yeast extract-treated cells of $A$. microcarpa, accompanying the change in the transcriptional activities.
\end{abstract}

Key words methyl jasmonate; calmodulin; gene expression; agarwood; Aquilaria microcarpa

Jasmonates, jasmonic acid and methyl jasmonate (MJ), are essential plant hormones that regulate defense responses against environmental stressors, such as drought, wounding, and microbial infection. In addition, exogenous application of jasmonates to plant cells enhance accumulation of a large number of secondary metabolites belonging to structurally diverse groups. ${ }^{1,2)}$ The jasmonate-mediated signal transduction pathway is intertwined with other hormonal pathways and positively or negatively alters the expression of a variety of target genes. Recent studies have demonstrated ${ }^{2,3)}$ that the active form of jasmonates is an amino acid-conjugate, jasmonoyl-isoleucine. This adduct functions through interaction with a protein complex that acts as the receptor of this plant hormone, ${ }^{1,3)}$ and this jasmonate-mediated signaling further binds to ubiquitin-proteasome-mediated protein degradation (Fig. 1). We have recently reported ${ }^{4,5}$ that biosynthesis of a tetracyclic diterpenoid of Scoparia dulcis, scopadulcic acid, is stimulated by the treatment of the plant with MJ, and that this process is triggered by a $\mathrm{Ca}^{2+}$-influx into the cytoplas-

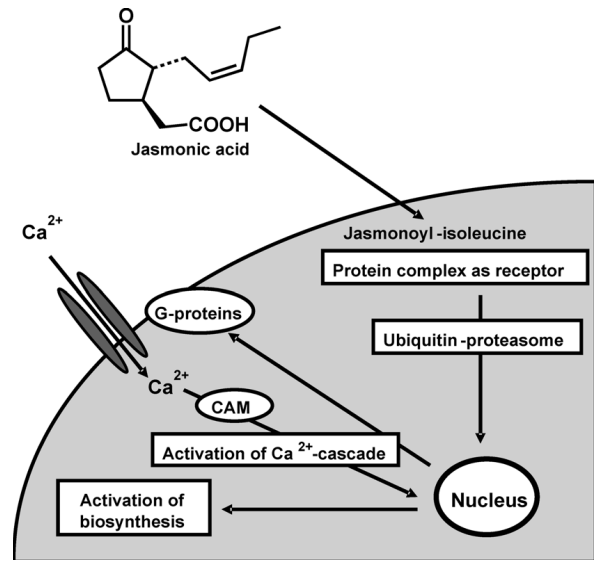

Fig. 1. Hypothetical Signal Transduction Processes in Jasmonate-Stimulated Plant Cells mic space of the cells. We also demonstrated that activation of a $\mathrm{Ca}^{2+}$-cascade in the signal transduction pathway is an essential requirement for MJ-induced diterpene production in S. dulcis (Fig. 1). However, very limited information is available on the detailed mechanisms by which jasmonates induce the biosynthesis of various secondary metabolites in plant cells.

Agarwood is generated in woody tissues of Aquilaria or some other plant species in response to either microbial infection or mechanical wounding, but is not generated under healthy conditions. ${ }^{6,7)}$ Although agarwood produces a variety of sesquiterpene compounds used as the scent, artificial transformation of agarwood-producing plants to agarwood has proved quite difficult. Okudera and Ito have recently reported $^{8)}$ that biosynthesis of a series of sesquiterpenoids, such as $\alpha$-guaiene, $\alpha$-humulene and $\delta$-guaiene, is induced in cell cultures of $A$. crassuna and $A$. sinensis by treatment with MJ.

In the present study, we established a callus culture of $A$. microcarpa, the tissues of which accumulate a unique spirovetivane-type sesquiterpene when the plant is transformed to agarwood. ${ }^{9)}$ Two cDNA clones presumably encoding calmodulin (CAM), a key mediator protein of the $\mathrm{Ca}^{2+}$ cascade in eukaryotic cells, ${ }^{10,11)}$ were isolated from the culture to investigate the biochemical and molecular biological processes of MJ-induced activation of the sesquiterpene biosynthesis in A. microcarpa. A possible change in the transcriptional activities of these genes in $A$. microcarpa cells in response to various external stimuli was also examined.

\section{MATERIALS AND METHODS}

Plant Material and Callus Induction A. microcarpa was grown in a green house at Experimental Station for Medicinal Plant Research in University of Toyama. Sterilized young leaf tissues of the plant were placed on Murashige and 
Skoog's agar medium ${ }^{12)}$ supplemented with $3 \mu \mathrm{M}$ of 2,4 dichlorophenoxyacetic acid (2,4-D), and callus formation was observed in the leaf veins after 4 weeks. The dedifferentiated cells were then incubated on the medium in the presence of $3 \mu \mathrm{M}$ of $2,4-\mathrm{D}$ and $3 \mu \mathrm{M}$ of $N^{6}$-benzyladenine at $26^{\circ} \mathrm{C}$ under darkness, and, after establishment of the callus culture, the cells were transferred onto a fresh medium every 4 weeks.

Cloning of Am-cam-1 and Am-cam-2 from A. microcarpa Total RNA was isolated from the callus culture of $A$. microcarpa with RNeasy Plant Mini Kit (Qiagen, Hilden, Germany), and cDNA templates were generated by reversetranscription (RT) reaction using Transcriptor First Strand cDNA Synthesis Kit (Roche, Basel, Switzerland). The cDNA fragments presumably encoding the translatable region of CAM gene(s) of $A$. microcarpa were isolated by polymerase chain reaction (PCR) amplification employing the primer pair, 5'-ATG GCC GAT CAG CTG ACG GAC GAC CAG ATC-3' as the forward and 5'-TYA CTT GGC CAT CAT AAC CTT GAC AAA YTC-3' as the reverse primer. The $3^{\prime}$ untranslatable region (UTR) of the CAM gene was obtained by means of $3^{\prime}$-rapid amplification of cDNA end (RACE) method using 5'/3' RACE Kit 2nd Generation (Roche). RT reaction was performed with an oligo dT-anchor primer, and PCR was carried out with 5'-GAT GTG GAT GGT GAT GGG CAG ATC AAC T-3' as the gene specific forward and PCR-anchor as the reverse primer. The amplified DNA fragments were subcloned into the pGEM-T vector (Promega, WI, U.S.A.), and the nucleotide sequences were determined on both strands using the dye-terminator method on a PRISM 3100 Genetic Analyzer (Applied Biosystems, CA, U.S.A.).

Over-Expression and Immunoblot Analysis Over-expression of Am-cam-1 and Am-cam-2 genes in Escherichia coli was performed using pBAD TOPO TA Expression Kit (Invitrogen, CA, U.S.A.) according to the instruction manual. The translatable regions of Am-cam-1 and Am-cam-2 were amplified by PCR and were subcloned into pBAD-TOPO. $E$. coli BL21/DE3 cells were transformed with the constructed expression vectors harboring the coding regions of $\mathrm{Am}$-cam genes. The cultures were grown at $37^{\circ} \mathrm{C}$ overnight, and then inoculated into $2 \mathrm{ml}$ of fresh medium. Arabinose (final concentration $0.002 \%, \mathrm{w} / \mathrm{v}$ ) was added to the cultures as the inducer when they reached an optical density of 0.6 at $590 \mathrm{~nm}$, and the incubation was further continued for $3 \mathrm{~h}$. The bacterial cells were harvested by centrifugation at $8000 \boldsymbol{g}$ for $5 \mathrm{~min}$, and the proteins were extracted by boiling the cells in $200 \mu$ of the sample buffer for sodium dodecyl sulfate-polyacrylamide gel electrophoresis (SDS-PAGE). The samples were subjected to SDS-PAGE (12\% gel), and the separated proteins were blotted onto a nitrocellulose membrane $(0.2 \mu \mathrm{m})$ on a semi-dry transfer cell (Bio-Rad, Transblot SD, U.S.A.). After blocking with $1 \%(\mathrm{w} / \mathrm{v})$ dry fat milk, the membrane was incubated with anti-CAM (Dictyostelium discoideum CAM, produced in mouse, Sigma-Aldrich, MO, U.S.A.) in $5 \mathrm{ml}$ Tris-buffered saline at $4{ }^{\circ} \mathrm{C}$ overnight. The membrane was then incubated with Protein $\mathrm{G}$ conjugated with horseradish peroxidase (Bio-Rad, CA, U.S.A.) at $25^{\circ} \mathrm{C}$ for $2 \mathrm{~h}$, and was washed several times with the buffered saline. Primary antibody bound to CAM protein was visualized by color development reaction (Bio-Rad).
RT-PCR Analysis of Am-cam-1 and Am-cam-2 Semiquantitative analysis of transcriptional activities of $A m-c a m-1$ and $A m-c a m-2$ was performed using RT-PCR. According to the method described previously, ${ }^{13)} 50 \mu$ l-aqueous solutions of MJ (100 $\mu \mathrm{M}$, Wako, Osaka, Japan), yeast extract (10 mg/ ml, Difco, MI, U.S.A.) or $\mathrm{Ca}^{2+}$-ionophore A23187 $(10 \mu \mathrm{M}$, Sigma) were added to cultured cells of $A$. microcarpa (approximately $100 \mathrm{mg}$ ), and control treatments received only water instead of these solutions. The cells were incubated in Petri dishes at $26^{\circ} \mathrm{C}$ under darkness, and at regular intervals, total RNA was isolated from the cells according to the method described above. Aliquots of RNA solutions (approximately $0.5 \mu \mathrm{g}$ RNA equivalent) were subjected to RT reaction, and then PCR was carried out with the appropriate primer pairs, 5'-GAT GTG GAT GGT GAT GGG CAG ATC AAC $\mathrm{T}-3^{\prime}$ as the common forward primer, and 5'-CAC CAA CAA GCC AAG CTT CTA GGG-3' for Am-cam-1 and 5'GAT CAG AAG CAA ATG AAA TCG C-3' for Am-cam-2 ( 270 mer and 298 mer as the products), as the gene specific reverse primers.

\section{RESULTS AND DISCUSSION}

Based on the reported amino acids sequences of plant CAMs, we isolated two cDNA $450 \mathrm{bp}$ clones presumably encoding the entire translatable region of the protein from $A$. microcarpa. The nucleotide sequences of these cDNA fragments were very similar, and only 6 nucleotides were found to be replaced. The putative amino acids sequences of the translational products of the two genes were identical (149 amino acid residues), and showed high homology with those of CAMs from various plant sources (Fig. 2). ${ }^{14-18)}$ They contained four highly conserved Asp-rich motifs characteristic of proteins that correspond to $\mathrm{Ca}^{2+}$-binding domains. To obtain more information about the structure of these CAM genes from $A$. microcarpa, we attempted to determine the nucleotide sequences of $3^{\prime}$-UTRs in the next experiment. Two cDNA fragments obtained by $3^{\prime}$-RACE method consisted of significantly different $3^{\prime}$-UTR structures, and these two gene regions including the CAM-coding region and $3^{\prime}$ UTR were designated as Am-cam-1 (450 plus $282 \mathrm{nu}$ cleotides, GenBank accession no. HM237343) and Am-cam2 (450 plus 251 nucleotides, GenBank accession no. HM237344), respectively. The translatable regions of these two genes were subcloned into pBAD-TOPO, and E. coli cells were transformed with the recombinant expression vectors. After the treatment of the cultures with arabinose, the proteins were extracted from the bacterial cells and were analyzed by SDS-PAGE (Fig. 3A). No obvious differences were observed between the control and the arabinose-induced samples after staining with Coomassie Brilliant Blue, however, the signals cross-reacted with anti-CAM were detected at the expected position (approximately $17 \mathrm{kDa}$ ) only in the arabinose-treated cells transformed with either Am-cam-1 or Am-cam-2 (Fig. 3B). These results strongly suggest that both Am-cam-1 and Am-cam-2 genes encode CAM protein in $A$. microcarpa.

The possible change in the transcriptional activity of $\mathrm{Am}$ cam-1 and Am-cam-2 in A. microcarpa cultures in response to various external stimuli was examined by RT-PCR (Fig. 4). Although the nucleotide sequences of the translational re- 
Am-cam-1, Am-cam-2 MADQLTDDQISEFKEAFSLFDKDGDGSITTKELGTVMRSLGQNPTEAELQDMINEVDADGNGT IDFPEFLNLMAR

\begin{tabular}{|c|c|}
\hline Poplar & $-C-$ \\
\hline Cedar & $--\mathrm{E}---\mathrm{E}---\mathrm{A}-\mathrm{-}-\mathrm{-}-\mathrm{-}-\mathrm{-}-\mathrm{-}-\mathrm{-}-\mathrm{C}-\mathrm{-}$ \\
\hline Arabidopsis-2 & --_--_- \\
\hline Carrot-11 & $--C-$ \\
\hline Alfalfa & 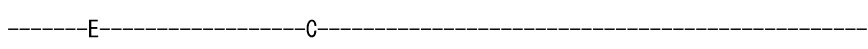 \\
\hline Barley & ----------A---------------C--- \\
\hline Potato-1 & - -E---EE--- \\
\hline
\end{tabular}

$\begin{array}{lllllll}80 & 90 & 100 & 110 & 120 & 130 & 140\end{array}$

Am-cam-1, Am-cam-2 KMKDTDSEEELKEAFRVFDKDQNGF ISAAELRHVMTNLGEKLTDEEVDEMVREADVDGDGQINYEEFVKVMMAK

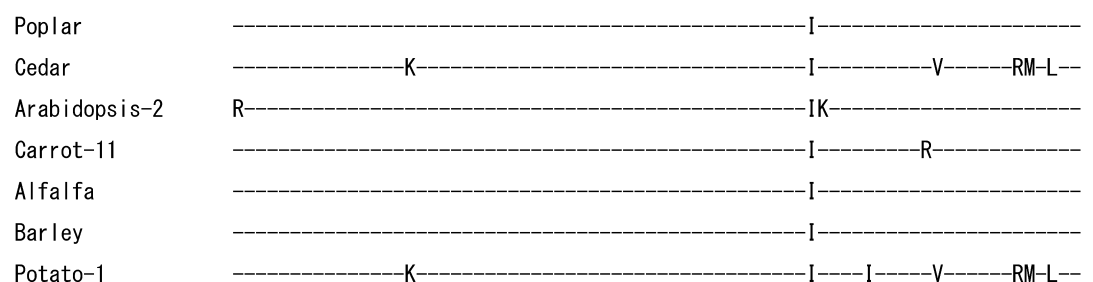

Fig. 2. Predicted Amino Acids Sequence of CAM Genes from A. microcarpa

Two CAM genes designated Am-cam- 1 and Am-cam-2 were isolated from callus culture of A. microcarpa. The deduced amino acid sequences of the translational products of these genes were identical, and were presented with those of CAM proteins from various plant sources (GenBank accession numbers; poplar, AAX63770; cedar, BAF32041; Ara-

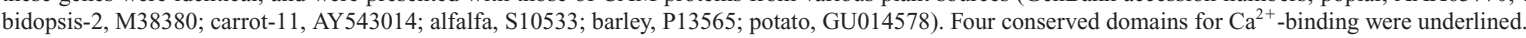

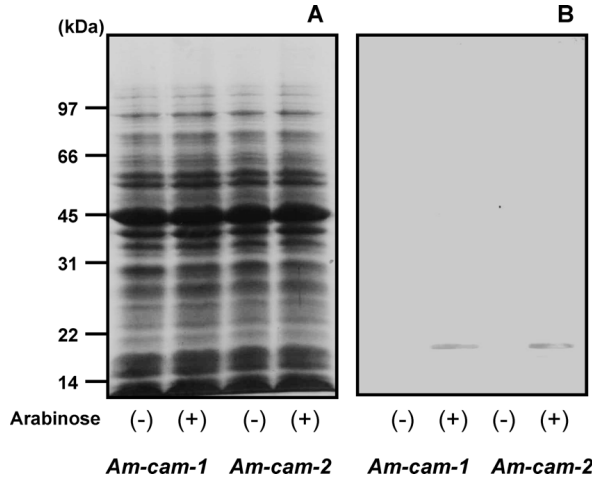

Fig. 3. Over-Expression of CAM Genes from A. microcarpa in E. coli Cells

Coding regions of Am-cam- 1 and Am-cam-2 were ligated into pBAD-TOPO, and $E$. coli cells were transformed by the constructed expression vectors. After the addition of arabinose as the inducer $(+)$ or $\mathrm{H}_{2} \mathrm{O}$ as the control $(-)$, the proteins were extracted from the cultures and were subjected to SDS-PAGE (A). The separated proteins were transferred onto a nitrocellulose membrane and were probed with anti-CAM (B).

gions of $A m-c a m-1$ and $A m-c a m-2$ were very similar, their $3^{\prime}$-UTR structures were quite different. We also confirmed that PCR performed with the combination of the common forward and gene specific reverse primers, as described above, yielded desired DNA fragments corresponding to the partial structures of Am-cam-1 and Am-cam-2. This allowed selective monitoring of the transcriptional activities of the two CAM genes in A. microcarpa under various conditions (Fig. 4). The expression activity of Am-cam-1 was maintained at a low level at time zero, however, it appreciably increased when the cell culture was treated with MJ. A marked increase in the gene transcription was observed after $3 \mathrm{~h}$ of treatment, and the enhanced activity continued for at least $24 \mathrm{~h}$. Expression of CAM gene, Am-Cam-2, was also stimulated by incubation with MJ. The intensity of the enhance-

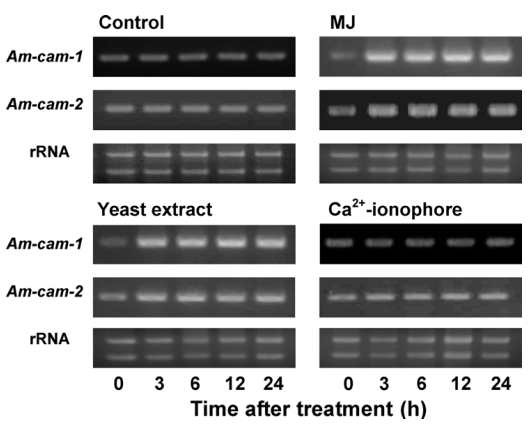

Fig. 4. Semi-Quantitative Analyses of the Expression of Am-cam-1 and Am-cam-2 in A. microcarpa by RT-PCR

Changes in the expression levels of Am-cam-1 and Am-cam-2 in callus culture of $A$. microcarpa were examined after the treatment with $\mathrm{MJ}$, yeast extract or $\mathrm{Ca}^{2+}$. ionophore A23187. The cultures were incubated with these stimuli, and at regular intervals, the cells were harvested and subjected to RT-PCR analyses. Control treatment received only water instead of these reagents.

ment, however, seemed to be appreciably lower than that of Am-cam-1. In the control treatment, no notable change in the expression activities was observed either in Am-cam-1 or Am-cam-2. A similar set of results was obtained for an A. $m i$ crocarpa culture treated with yeast extract that has been known to induce biosynthesis of various types of secondary metabolites in many plant species. ${ }^{19,20)}$ The transcriptional activity of Am-cam-1 was greatly enhanced in yeast extract treated cultures, however, the level of induced activation of Am-cam-2 expression was noted to be somewhat lower than that in Am-cam-1. In sharp contrast to incubation with MJ and yeast extract, the expression of both Am-cam-1 and Amcam-2 showed only a negligible change, if any, on incubation with $\mathrm{Ca}^{2+}$-ionophore $\mathrm{A} 23187$, and the transcription of these two CAM genes was maintained at an almost constant level over the testing procedure. In repeated experiments, a similar set of results was reproducibly observed, though the relative 
levels of the expression activities of Am-cam-1 and Am-cam-

2 varied to some extent.

In the present study, we isolated two cDNA clones, $\mathrm{Am}$ cam-1 and Am-cam-2, as CAM genes from tissue cultures of A. microcarpa (Fig. 2). The expression levels of both Amcam-1 and Am-cam-2 increased in response to stimulation with either MJ or yeast extract, suggesting that these two CAM genes and their products play important roles in the signal transduction processes accompanying the change in the transcriptional activities when the cells are stimulated with these solutions. We have reported previously ${ }^{5)}$ that CAM functions as the key mediator protein in MJ-induced diterpene accumulation in $S$. dulcis. The results obtained in the present study are, therefore, consistent with our previous finding that activation of $\mathrm{Ca}^{2+}$-cascade is prerequisite for the enhancement of secondary metabolites biosynthesis triggered by $\mathrm{MJ}$ in plant cells. Although expression activities of both Am-cam-1 and Am-cam-2 respond positively to stimulation by $\mathrm{MJ}$ and yeast extract, it is likely that $A m-c a m-1$ is more sensitive to such external stimuli (Fig. 4). A series of recent studies $^{18,21,22)}$ has demonstrated that plant CAMs, unlike in animals, are composed of several isoforms and the expression of specific CAM genes is sometimes induced by appropriate stimuli or environmental stressors. The different responses of the two CAM genes of $A$. microcarpa to various external stimuli would be, therefore, in agreement with the hypothetical properties of plant CAMs. The expression levels of Am-cam-1 and Am-cam-2 appreciably increased in the presence of $\mathrm{MJ}$ and yeast extract, but not in the presence of $\mathrm{Ca}^{2+}$-ionophore A23187 (Fig. 4). This 'selective sensitivity' of Am-cam-1 and Am-cam-2, i.e., increased sensitivity to the primary stimuli (MJ and yeast extract) but negligible sensitivity to $\mathrm{Ca}^{2+}$-ionophore $\mathrm{A} 23187$, leads us to assume that the induced expression of these two CAM genes might be evoked by a certain signal generated by $\mathrm{MJ}$ reception, but not by the increased concentration of cytoplasmic $\mathrm{Ca}^{2+}$ (Fig. 1). However, at present, it is not clear whether or not the apparent insensitivity of CAM genes transcription toward $\mathrm{Ca}^{2+}$-influx is shared as the common mechanism in a wide range of plant species upon the stimulation with MJ.

Further studies on the biochemical and molecular biological mechanisms of MJ-induced activation of secondary me- tabolism in $A$. microcarpa are in progress in our laboratory.

Acknowledgments The authors should appreciate Professor S. Kadota, Institute of Natural Medicines, University of Toyama, providing the seeds of A. microcarpa. The authors also thank Y. Takao, Y. Tatsuo and H. Fujino, Experimental Station for Medicinal Plant Research in University of Toyama, for cultivation of the plant.

\section{REFERENCES}

1) Browse J., Annu. Rev. Plant Biol., 60, 183-205 (2009).

2) Katsir L., Chung H. S., Koo A. J., Howe G. A., Curr. Opin. Plant Biol., 11, $428-435$ (2008).

3) Turner J. G., Ellis C., Devoto A., Plant Cell, 14, S153-S164 (2002).

4) Hayashi T., Okamura K., Kawasaki M., Morita N., Phytochemistry, 33, 353-356 (1993).

5) Nkembo K. M., Kurosaki F., Lee J. B., Hayashi T., Plant Biotechnol., 22, 333-337 (2005).

6) Ito M., Honda G., Nat. Med., 59, 104-112 (2005).

7) Ng L. T., Chang Y. S., Kadir A. A., J. Forest. Prod., 2, 272-285 (1997).

8) Okudera Y., Ito M., Plant Biotechnol., 26, 307-315 (2009).

9) Ueda J., Imamura L., Tezuka Y., Tran Q. L., Tsuda M., Kadota S., Bioorg. Med. Chem., 14, 3571-3574 (2006).

10) Krestsinger R. H., CRC Crit. Rev. Biochem., 8, 119-174 (1980).

11) Klee C. B., Vanaman T. C., Adv. Protein Chem., 35, 213-321 (1982).

12) Murashige T., Skoog F., Physiol. Plant, 15, $473-479$ (1962).

13) Shite M., Yamamura Y., Kurosaki F., Plant Biotechnol., 26, 403-408 (2009).

14) Ishigaki E., Sugiyama R., Kurosaki F., Biol. Pharm. Bull., 28, 1109 1112 (2005).

15) Ling V., Perera I., Zielinski R. E., Plant Physiol., 96, 1196-1202 (1991).

16) Barnett M. J., Long S. R., Nucleic Acids Res., 18, 3395 (1990)

17) Ling V., Zielinski R. E., Plant Physiol., 90, 714-719 (1989).

18) Takezawa D., Liu Z. H., An G., Poovaiah B. W., Plant Mol. Biol., 27, 693-703 (1995).

19) Zhao J., Davis L. C., Verpoorte R., Biotechnol. Adv., 23, 283-333 (2005).

20) Gundlach H., Muller M. J., Kutchan T. M., Zenk M. H., Proc. Natl. Acad. Sci. U.S.A., 89, 2389-2393 (1992).

21) Heo W. D., Lee S. H., Kim M. C., Kim J. C., Chung W. S., Chun H. J., Lee K. J., Park C. Y., Park H. C., Choi J. Y., Cho M. J., Proc. Natl. Acad. Sci. U.S.A., 96, 766-771 (1999).

22) Yamatake H., Mitsuhara I., Ito S., Seo H., Kamada S., Ohashi Y., Eur. J. Biochem., 268, 3916-3929 (2001). 